\title{
COMMUNICATIONS
}

\section{RECORDING OF THE OCCIPITAL LOBE RESPONSE IN MAN AFTER LIGHT STIMULATION*}

\author{
BY
}

\author{
A. T. M. VAN BALEN AND H. E. HENKES \\ Eye Clinic, Rotterdam
}

THE discovery by Du Bois-Reymond (1849), of electrical changes in the neuron during the passage of a stimulus, was the starting point for the objective functional investigation of the nervous system.

The electrophysiological experiments on the retina based on this discovery led to the establishment of electroretinography and its clinical use.

The optic nerve, the optic tract, the lateral geniculate body, and the optic radiation are not yet accessible to objective clinical investigation. The optic cortex and the subcortical structures, on the other hand, are accessible for objective investigation through electro-encephalography; the electroencephalogram is used to demonstrate clinically the effect of intermittent light stimuli on the spontaneous activity of the occipital cortex.

In his first experiments, Berger (1931) was able to demonstrate the influence of opening the eyes and of a light stimulus on the spontaneous activity of the occipital region in a subject placed in a darkened room. He observed an interruption of the electrical activity, the so-called blocking of the alpharhythm. Fig. 1 serves as an example.

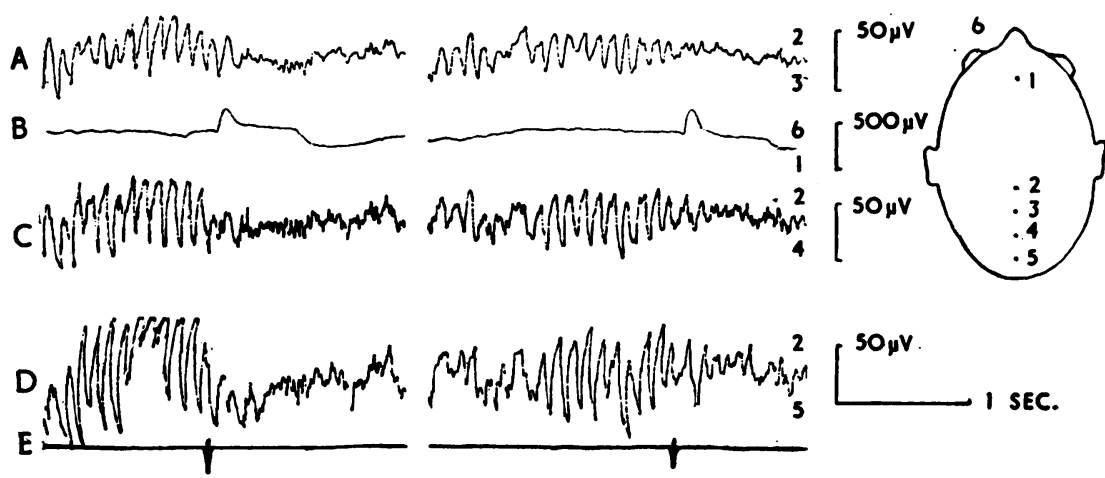

Fig. 1.-Blocking of alpha-rhythm in electro-encephalogram recordings (Tracings A, C, and D) by light stimulus (Tracing E). Channel B records the scotopic electroretinogram of the left eye. Channels A, C, and D record the electro-encephalogram using electrodes placed in the mid-line of the occipital region. Indifferent electrode (Number 1) placed on forehead. Number 6: contact lens electrode. Time constant $0.7 \mathrm{sec}$.

* Received for publication August 21, 1959. 449 
Kluyskens (1948) made use of this "alpha-blocking" in the objective determination of the visual field. The alpha-blocking, however, is by no means a specific reaction. It can be elicited by any type of afferent impulse: sound, touch, etc. Moreover, its latency is long and extremely variable $(150-300 \mathrm{msec}$.$) . The reaction depends upon the power of concentration and$ the emotional state of the subject. Repeated stimulation may sometimes fail to elicit the blocking reaction. Finally, a serious disadvantage is that a fixation light cannot be used during the experiment, as fixation in itself blocks the alpha-activity.

The specific or "evoked" response of the striate area and optic cortex after a light stimulus is in our opinion more deserving of the ophthalmologist's attention. Unfortunately, the evoked response of the occipital lobe is recordable in routine electro-encephalography in only a small percentage of subjects. Monnier (1956) found a perceptible response in only a few subjects, and François, Stefens, and de Rouck (1954) claimed a percentage of twenty in normal subjects. Fig. 2 gives an example of a clearly recordable evoked response, but in Fig. 3 the specific response is not discernible owing to the high spontaneous activity of the cortex and subcortical structures.

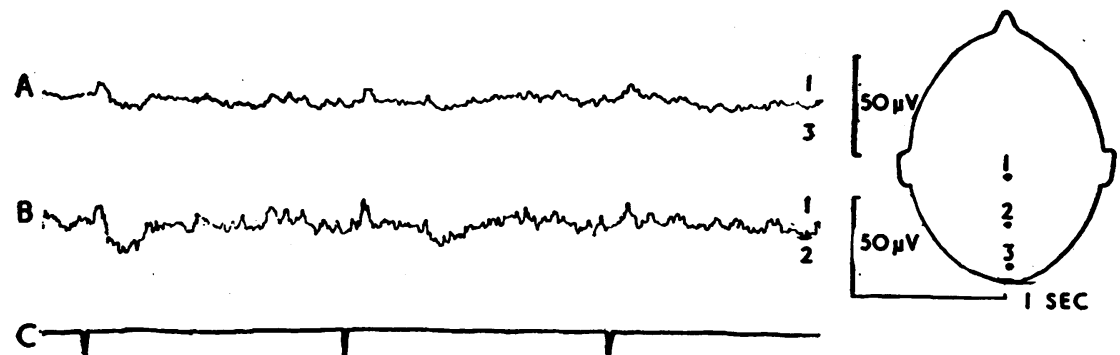

FIG. 2.-Clearly visible evoked responses after light stimulation (Tracing C). Records taken from electrodes placed in the midline of the occipital region (Tracings $A$ and $B$ ).

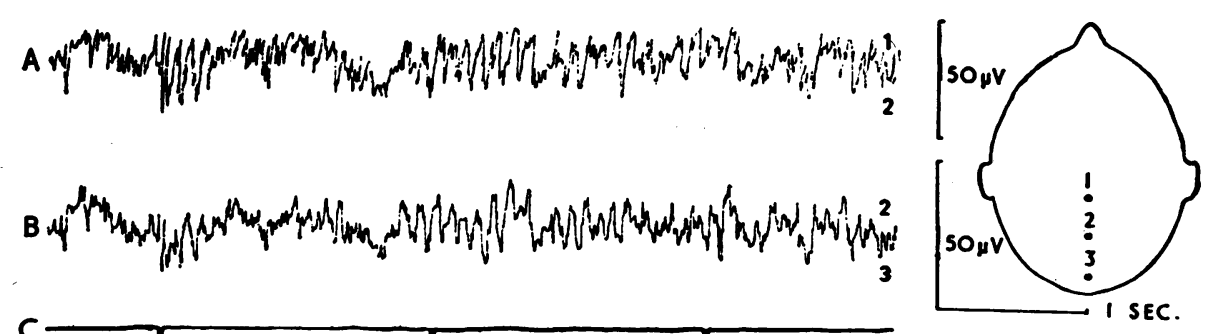

FIG. 3.-No discernible responses in records (A, B) taken from electrodes placed in the midline of the occipital region. Light stimulus on Channel $\mathrm{C}$. 
Before the recording of the occipital lobe response is accepted as a routine method in the clinical investigation of the visual system, it is necessary to develop recording techniques which allow a positive result in all normal subjects examined.

\section{Recording Technique of the Evoked Response of the Occipital Lobe}

In cases in which the evoked response to a light stimulus is not clearly visible, one may use several methods to make the specific response recognizable among the random fluctuations of spontaneous activity.

Monnier, for instance, superimposed five recordings by drawing an enlarged projection. This method gives satisfactory results in only a small number of cases (as an example: see Fig. 4). An improved method of recording is that introduced by Dawson (1950). This involves the superimposition

FIG. 4.-Superimposition of five electroretinographic records (upper tracings), and five electro-encephalographic records from occipital region (lower tracings) in order to detect specific responses. Arrow indicates light stimulus.

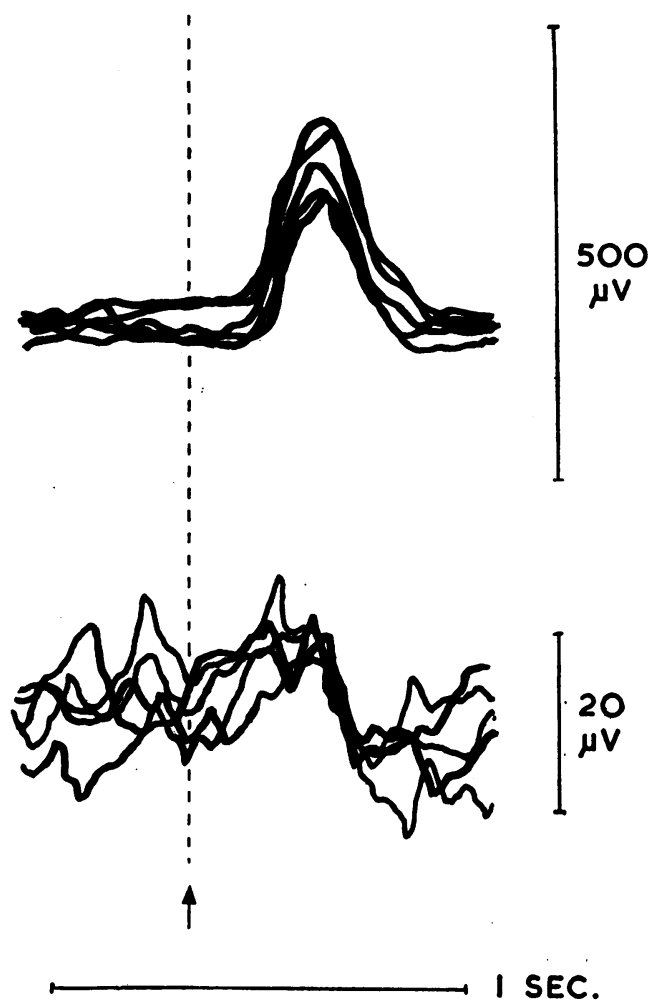

of a large number of sweeps of the cathode-ray oscilloscope, each sweep being triggered by the light stimulus itself and tracing the occipital lobe response belonging to this stimulus. About fifty successive electro-encephalograms are recorded on a stationary photographic film. This method allows a more exact superimposition of the individual electro-encephalographic records than can be got by copying and superimposing the projected curves. 
Features occurring at a constant interval of time after the stimulus appear more clearly, while those not related to the stimulus produce only a general broadening of the curve. This broadening of the build-up curve interferes with the measurement of amplitudes and latencies of the components present, although the response in itself becomes visible in the majority of subjects. Fig. 5 illustrates the usefulness of this method in a normal subject, and Fig. 6 shows in another normal subject that the presence of a specific response is only indicated.

FIG. 5.-Dawson's method of superimposing fifty separate records, triggered by the light stimulus, produces a clear picture of evoked occipital lobe response in a human subject: $b$-, $c$-wave complex, and $d$-wave are well marked. White vertical line on right side of record represents a calibration of $20 \mu \mathrm{v}$. Time constant $0.7 \mathrm{sec}$. Time base 50 cps.
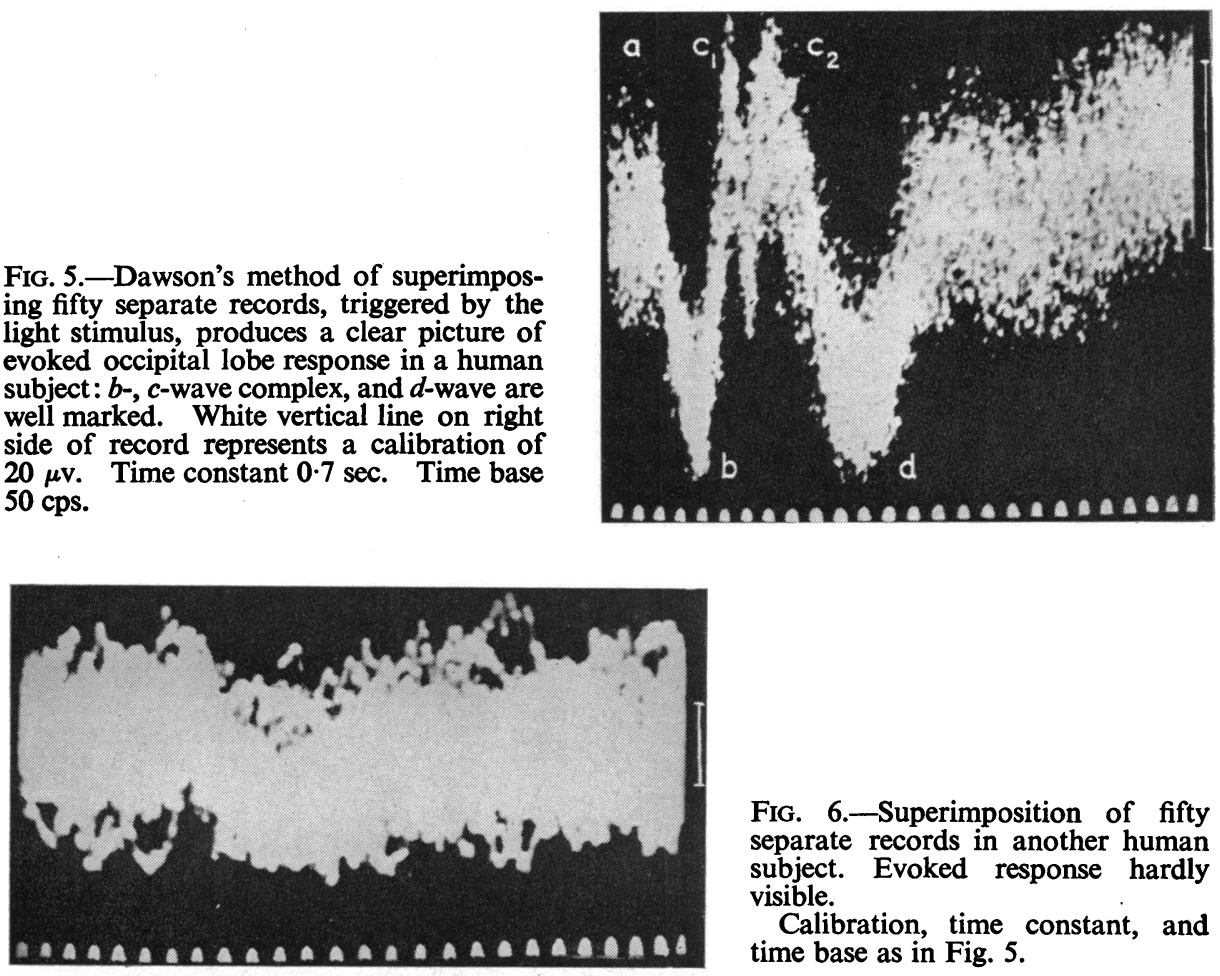

FIG. 6.-Superimposition of fifty separate records in another human subject. Evoked response hardly visible.

Calibration, time constant, and time base as in Fig. 5.

The most attractive method is the estimation of the mathematical average of a number of records. This technique allows the highest increase in the signal-to-noise ratio, making the evoked occipital lobe response recordable in all normal human subjects. The accuracy depends on the number of integrated separate records and on the number of, and distance between, the points over a tracing of a given length. Fig. 7 (opposite) gives the integration curve together with the superimposed curve obtained by recording forty separate responses. For the construction of the integration curve forty points on forty curves have been integrated, divided over a tracing of a length of $500 \mathrm{msec}$. This cipher method is extremely time-consuming, the construction of one curve taking approximately 10 hours' work. 
FIG. 7.-Mathematical integration curve based on forty different records drawn upon the superimposition figure of the same number of occipital lobe responses. Following the specific response, three waves are visible exhibiting the same frequency as the alpha-rhythm (1012 cps.).

Calibration, time base, and time constant as in Fig. 5.

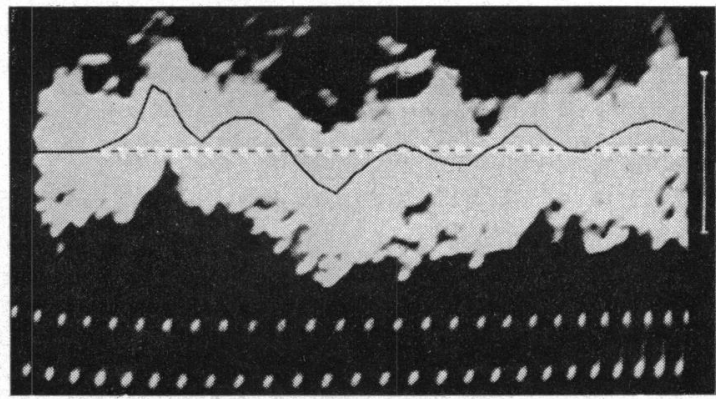

An electronic toposcope, such as that constructed by Dawson (1954), does this work within a few seconds. For the future development of the routine recording of occipital lobe responses in the ophthalmological clinic, the use of simplified electronic toposcopes is in our opinion indispensable.

In the present study the integration technique was used only in cases in which the information from the superimposed curves regarding the latency time of the different components of the response was insufficient.

Fig. 8 demonstrates the specificity of the response in regard to the visual stimulation. An electronic stroboscope served as a light stimulus. The superimposed image of the evoked response is shown in Fig. 8B. The stimulus source gives a click while delivering the lightflash. If the strobo-

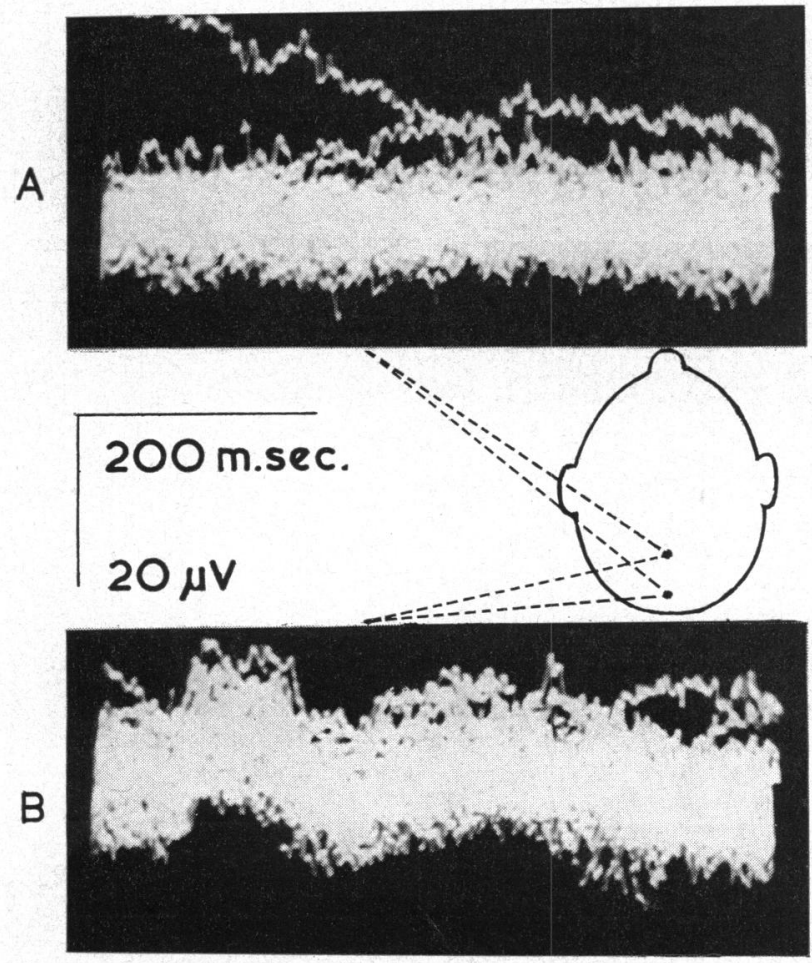
scope light is covered but the click continues to give an auditive stimulus no response is produced (see Fig. 8A).

Fig. 8.-Forty superimposed records of occipital lobe electro-encephalograms.

A. Light-tight covered stroboscope produces click sound. Auditive stimulation is unable to evoke response.

B. Stroboscope uncovered.

The evoked response is clearly visible. 
The recording of the evoked response on light stimulation is limited, generally speaking, to the occipital region. Fig. 9 shows the superimposed recordings of a number of leads from the temporal, parietal, and occipital areas. The evoked response is found mainly over the occipital lobe, and the same stimulus does not normally elicit a marked response over the other areas mentioned.

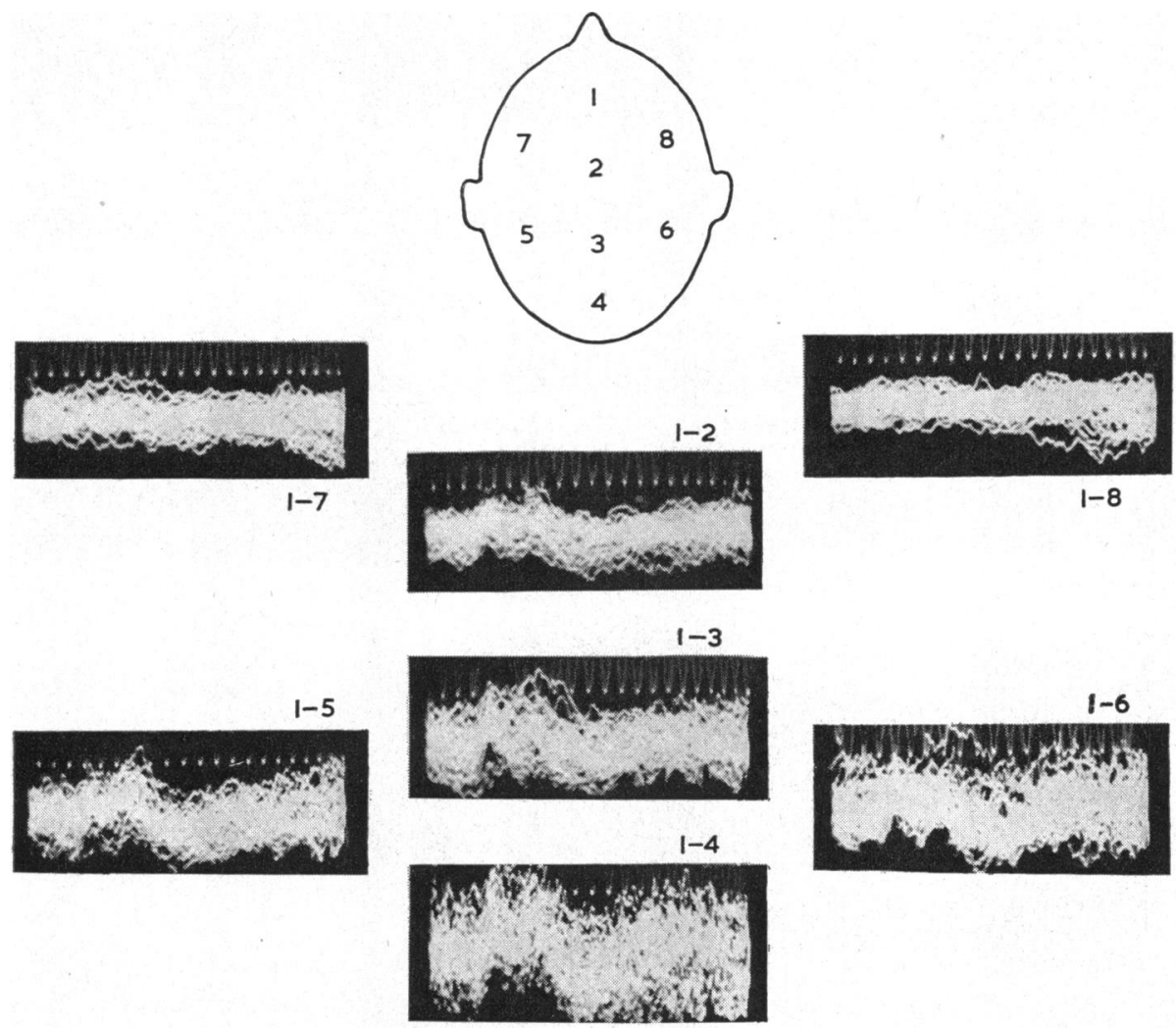

Fig. 9.-Forty superimposed records of responses led from Electrodes 2, 3, 4, 5, and 6. Parietal electrodes 7 and 8 are unable to record specific response. Electrode 1 on forehead serves as a reference.

\section{Components of the Evoked Potential}

In our own series of normal patients, the latencies of the components of the occipital lobe response closely resemble those found by Monnier and by François, at least if we take into account the different conditions of stimulation. Monnier distinguishes three main deflections: the $b$-, $c$-, and $d$-waves (Fig. 5)*.

* Regarding the polarity of the waves concerned, the denomination of Monnier (1956) has been followed. Probably, however, it would be more logical to consider the $b$-wave as a negative, rather than a positive wave, and the $c$-wave complex as a positive complex, etc., as in our experiments the $b$-wave is the expression of the fact that the occipital electrode becomes negative in relation to a more forward placed electrode $(e . g$. Electrodes 4 and 3 , versus 2 and 1 , in Fig. 9). 
In our own subjects we found the following average values for different deflections:

\begin{tabular}{|c|c|c|c|c|}
\hline Deflection & & & Latency & Culmination Time (msec.) \\
\hline $\begin{array}{l}\text { First Positive ( } b \text {-wave) .. } \\
\text { First Negative ( } c \text {-wave complex) } \\
\text { Second Positive ( } d \text {-wave) }\end{array}$ & & $\begin{array}{l}\cdots \\
\cdots \\
\cdots\end{array}$ & $\begin{array}{c}40-50 \\
-\end{array}$ & $\begin{array}{l}65-75 \\
100-140 \\
180-220\end{array}$ \\
\hline
\end{tabular}

In a number of cases the specific response is followed by a series of waves which have the same frequency as the alpha-rhythm: 10 to 12 cycles per second (cps). Fig. 7 gives an example of this phenomenon.

The $b$-wave of the evoked response is present only in a limited number of subjects. The $c$-wave, on the other hand, is found in the vast majority of cases and may be considered as the most constant response.

Under special conditions of light stimulation, the $c$-wave presents a double top $\left(c^{1}, c^{2}\right.$, see Fig. 5). This phenomenon has already been described by van Hof (1959).

\section{Relationship between Electroretinography and the Evoked Potential}

This duplication of the $c$-wave may readily suggest to an ophthalmologist's mind the idea of a separate representation of photopic and scotopic activity. A correlation between the first culmination of the $c$-wave $\left(c^{1}\right.$, culmination time $100 \mathrm{msec}$.) and photopic activity, as well as a correlation between the second culmination ( $c^{2}$, culmination time $140 \mathrm{msec}$.) and scotopic activity, is provided by the records found in two different subjects (Figs 10 and 11, overleaf). Only $c^{2}$ is found in dark adaptation if the subject is stimulated with an extremely low-intensity flash which does not interfere with the state of adaptation (Fig. 10A, and Fig. 11A, lower tracing). When the subject is stimulated with a high-intensity flash in light adaptation, a preceding negative deflection $\left(c^{1}\right)$ comes into being in the evoked potential of the occipital lobe response (Fig. 10B, and Fig. 11B, lower tracing).

We are able to follow the changes in the evoked potential in the course of the process of dark adaptation. Fig. 12 (overleaf) illustrates such an experiment, in which the occipital lobe response was recorded after preliminary light adaptation to 1,750 asb. (Goldmann-Weekers adaptometer). The recording was repeated at different levels of dark adaptation, as given by the points A, B, C, and D. A very low intensity of the stimulus light was used in these experiments in order not to interfere with the adaptation process. In the successive stages of dark adaptation, a shift in the culmination time of the $c$-wave complex was observed. Owing to the appearance of $c^{2}$, the culmination time of the $c$-wave complex lengthened in the course of dark adaptation (visible only on the integrated curves).

In those cases in which the activity of the scotopic retinal system has been lost (e.g. cases of primary pigmentary degeneration of the retina) - in which, 


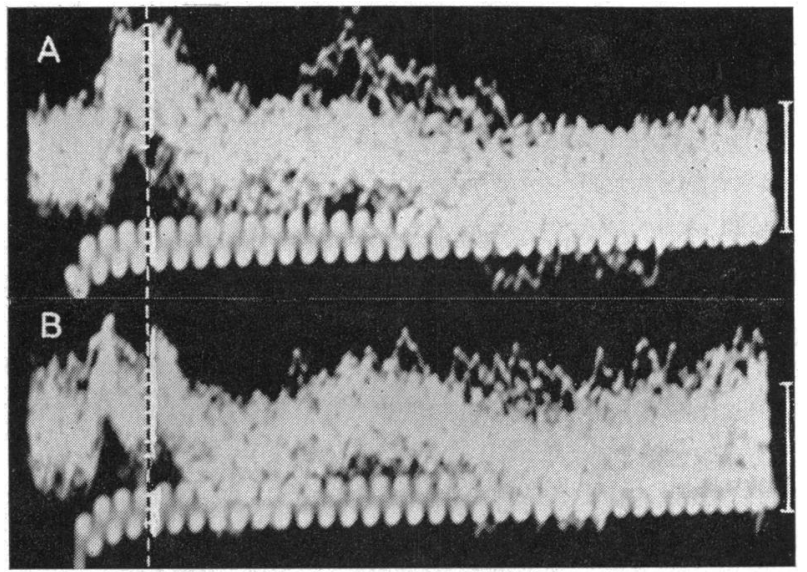

Fig. 10.

A. Superimposed records of evoked response after low-intensity flashes in dark adaptation. B. The same after high-intensity flashes in light adaptation.

Reduction in latency of $c$-wave complex (Fig. 10B) is due to the occurrence of preceding $\left(c^{1}\right)$ top. Dotted line marks $125 \mathrm{msec}$. after stimulus. Calibration $20 \mu \mathrm{v}$. Time base $50 \mathrm{cps}$.

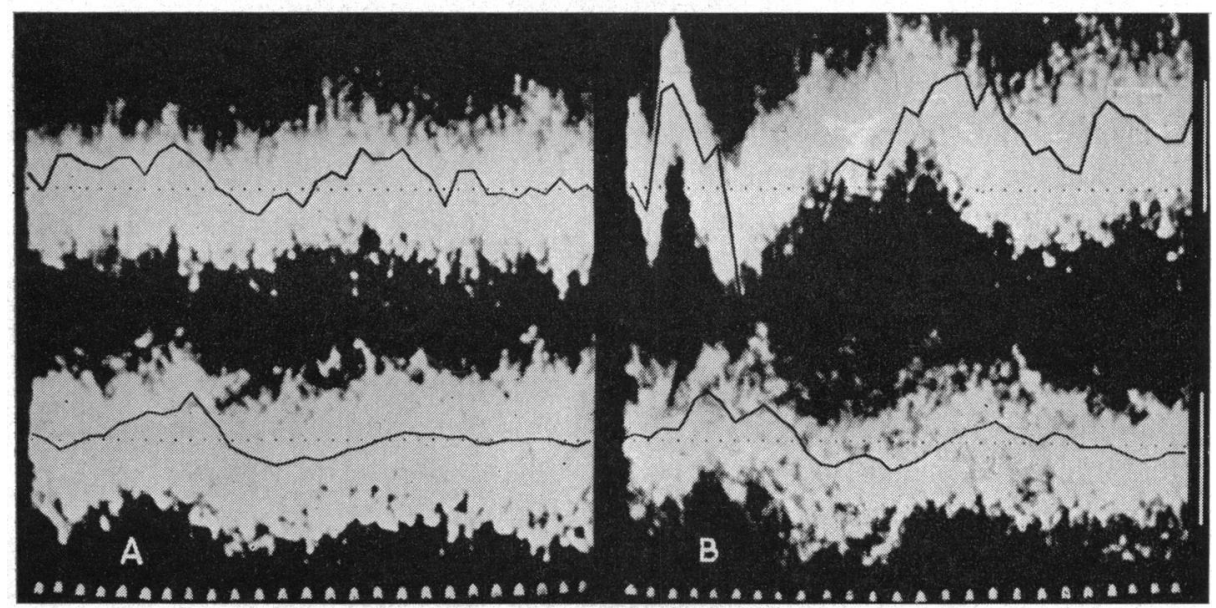

FIG. 11.-Superimposed records combined with mathematical integration curves of forty electroretinogram (upper tracings) and forty electro-encephalogram (lower tracings) responses. In dark adaptation (A) after low-intensity flashes, the presence of a scotopic electroretinogram response is doubtful, notwithstanding integration of responses. The $c$-wave complex in the occipital lobe response (lower tracing) is represented only by a late top $\left(c^{2}\right)$. In light adaptation (B) after highintensity flashes, a clear photopic electroretinogram is visible (upper tracing). The $c$-wave complex in the occipital lobe response (lower tracing) shows a double top ( $c^{1}$ and $c^{2}$ ). White vertical lines represent calibration of $20 \mu \mathrm{v}$. Time base $50 \mathrm{cps}$.

at best, only a rudimentary photopic retinal response can be recorded-only the $c^{1}$-wave of the evoked potential can be recorded after a stimulus which, in the normal subject, would elicit both $c^{1}$ and $c^{2}$. Fig. 13 (opposite) shows the evoked response of the occipital lobe in such a case. Under "normal" conditions of recording of the electroretinogram, the electrical response of the 

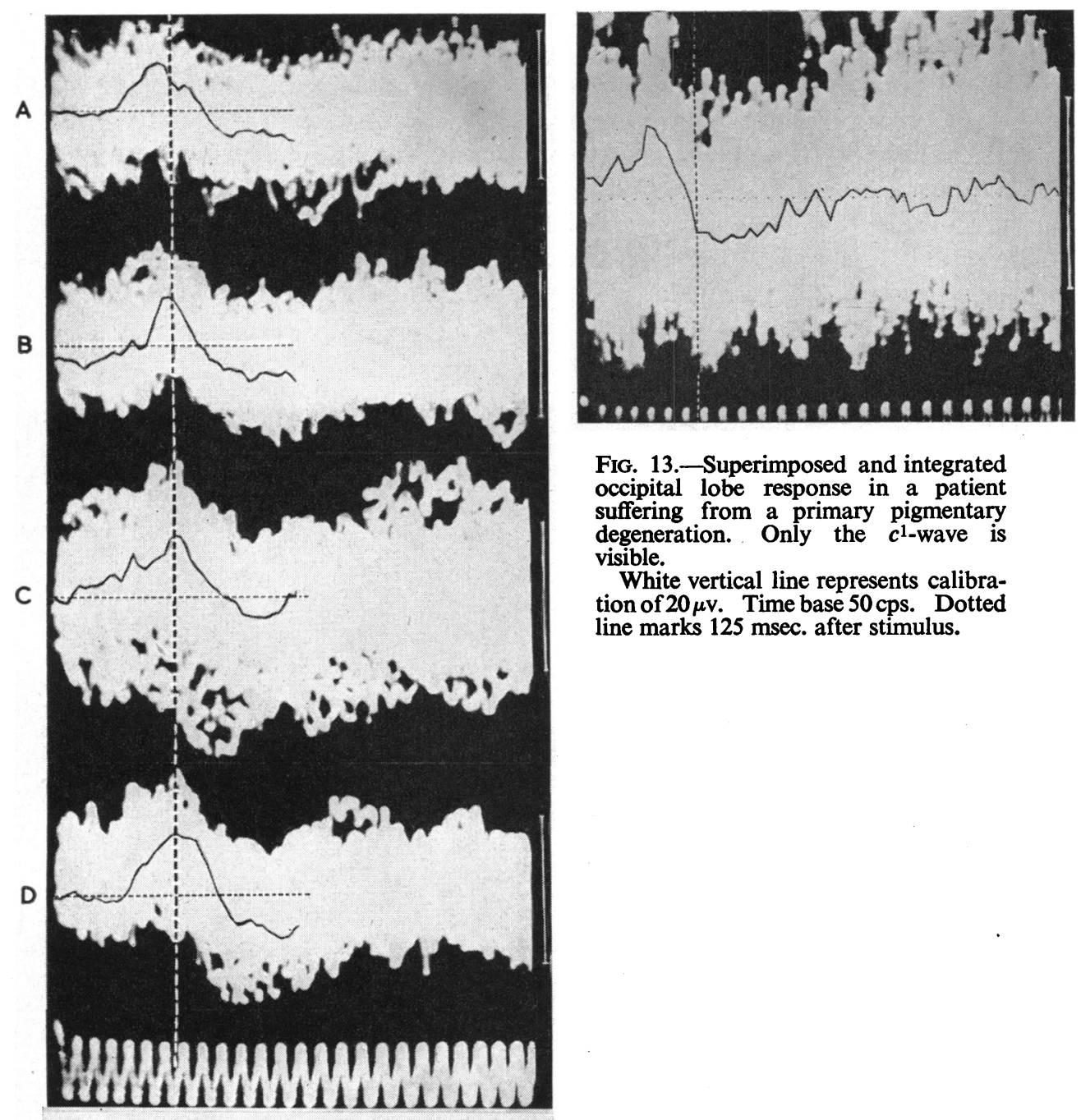

FIG. 13.-Superimposed and integrated occipital lobe response in a patient suffering from a primary pigmentary degeneration. Only the $c^{1}$-wave is visible.

White vertical line represents calibration of $20 \mu \mathrm{v}$. Time base $50 \mathrm{cps}$. Dotted line marks $125 \mathrm{msec}$. after stimulus.

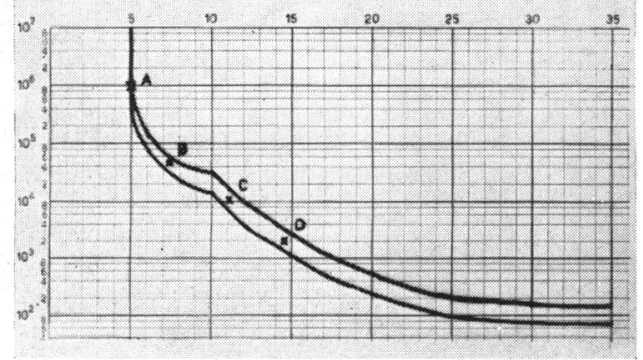

FIG. 12.- Superimposed and integrated curves of occipital lobe responses in the course of dark adaptation (GoldmannWeekers adaptometer). Tracings represent evoked responses at succeeding levels of dark adaptation (A, B, C, and D). Note shifting of culmination of the $c$-wave in relation to dotted line. White lines represent calibration of $20 \mu \mathrm{v}$. Time base $50 \mathrm{cps}$. . 
retina was extinguished, though selective amplification (Henkes, 1956) made a rudimentary photopic response recordable. In this case the visual field was limited to $10^{\circ}$ centrally. The occipital lobe response consisted of the $c^{1}$-wave only, and was clearly visible only in the integration curve.

A recording of the evoked potential may be important in the study of the optic pathways, for instance in the examination of cases suffering from unilateral optic atrophy. In such cases, the electroretinogram may be of normal size on the affected side, thus demonstrating objectively a normal function of the retina (Fig. 14A, lower tracing). The occipital lobe response, however, may be absent if the affected eye is stimulated alone (Fig. 14B, lower tracing).

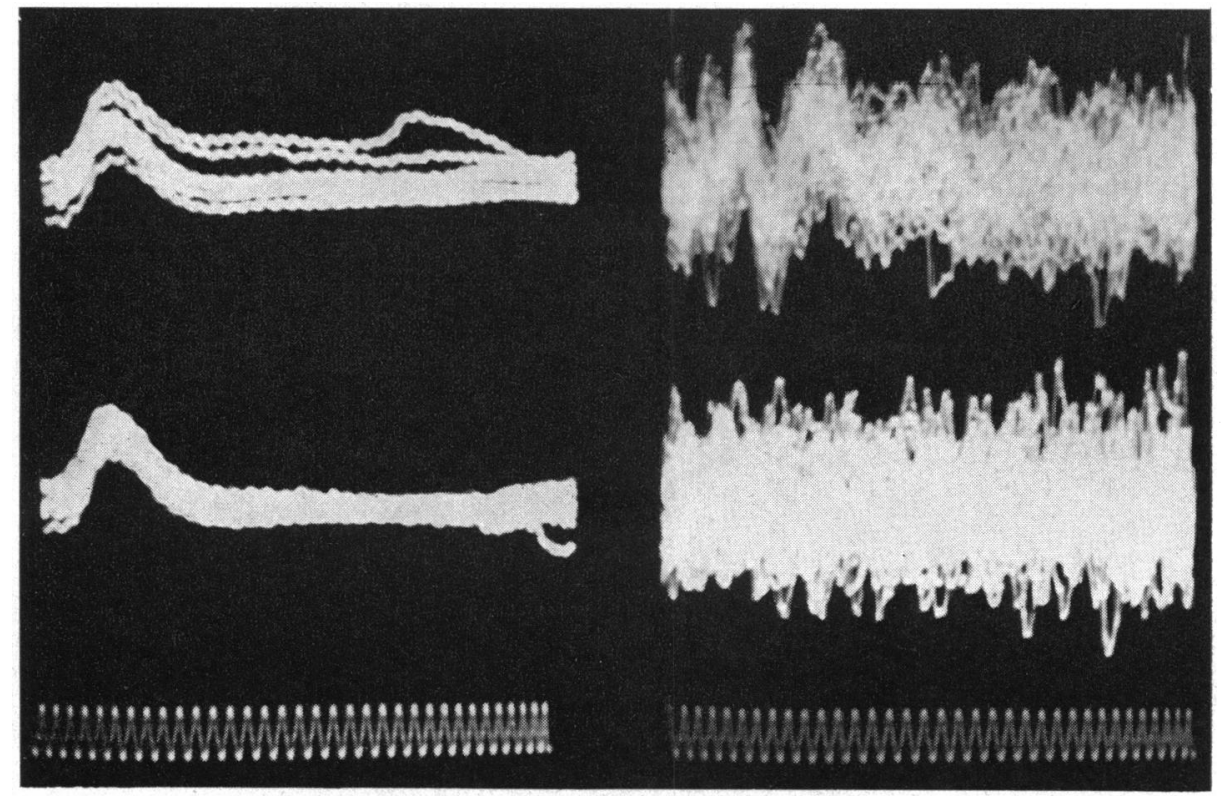

A

B

FIG. 14.-Superimposed electroretinographic (A) and electro-encephalographic (B) responses of right eye (upper tracings) and left eye (lower tracings) in a case of complete secondary atrophy of the left optic nerve. Absence of electro-encephalographic response (lower tracing, B) on stimulation of left eye, notwithstanding normal electroretinogram (lower tracing, A). Electroretinogram and electro-encephalogram of right eye are both normal (upper tracings). Time base $50 \mathrm{cps}$.

\section{Discussion}

In the study of the evoked potentials of the optic cortex in man, it is necessary -in order to be able to record the specific response in all normal subjects-to improve the signal-to-noise ratio by integrating a great number of 
separate responses. Mathematical averaging is the method of choice; without the use of modern electronic devices, however, this technique is extremely time-consuming.

Although the evoked occipital lobe response is triggered by an impulse starting in the retina, the amplitude of the components of this specific occipital response seems only partially dependent on the height of the potential of the different electroretinogram components. The amplitude of the evoked potential seems to depend, at least partially, on the electrical properties of the subcortical centres and the cortex itself as they are at the moment the impulse arrives in the (sub)cortical structures.

The experiments described above point towards the existence of a system, exhibiting a duplex nature, which gives rise to the evoked potential of the occipital cortex. It is very probable that the $c^{1}$-and the $c^{2}$-waves represent the photopic and scotopic activity of the retina.

Jayle, Boyer, and Camo (1958) published a preliminary note on the correlation between changes in the electroretinogram and the occipital lobe response in the course of the process of dark adaptation. In the occipital response they observed the development of a second $c$-wave, recordable only after a period of at least 5 minutes of dark adaptation. Bartley (1942) observed a double "on" response in the optic nerve of the rabbit, suggesting a separate representation of the retinal cone and rod systems. The difference in latency of the peaks was found to be $30 \mathrm{msec}$. This time lapse closely resembles the time interval found by us between the two peaks $c^{1}$ and $c^{2}$ in the occipital lobe response in man.

The possibility of differentiating between the scotopic and photopic mechanisms in the occipital lobe response, as well as that of demonstrating changes in the electrical activity of the visual pathways based upon pathological alterations, suggest that in the future the recording of the occipital lobe response may become a routine procedure in clinical ophthalmology.

\section{Summary}

The authors describe the so-called "primary" or evoked potential of the occipital lobe after light stimulation in normal human subjects. Several methods devised to improve the signal-to-noise ratio in the occipital lobe response are discussed.

The mathematical averaging of a great number of records (usually forty) seems to be the method of choice, enabling a specific response to be recorded in all normal human subjects. It is demonstrated that the photopic and scotopic mechanisms are each represented separately in the occipital lobe response.

Suggestions are given for using this recording technique in the investigation of the visual pathways in clinical ophthalmology. 


\section{REFERENCES}

Bartley, S. H. (1942). J. exp. Psychol., 30, 125.

BERGER, H. (1931). Arch. Psychiat. (Berl.), 94, 16.

Dawson, G. D. (1950). Brit. med. Bull., 6, 326.

(1954). Electroenceph. clin. neurophysiol., 6, 65.

DU Bois-ReYMOND, E. (1849). "Untersuchungen über thierische Elektricität", vol. 2, pt. 1, p. 256. Reimer, Berlin.

François, J., Strfens, R., and de Rouck, A. (1954). Ann. Oculist. (Paris), 187, 45.

HENKES, H. E. (1956). Bibl. ophthal., 48, 125.

HOF, M. W. VAN (1959). Electroenceph. clin. neurophysiol., 11, 611.

JAYLE, G. E., BOYER, R., and CAMO, R. (1958). C. R. Soc. Biol. (Paris), 152, 1725.

KLUYSKens, J. (1948). Bull. Soc. belge Ophtal., No. 90, p. 518. MONNIER, M. (1956). Bibl. ophthal., 48, 15. 